\title{
PLANEJAMENTO COMPOSTO CENTRAL APLICADO A SISTEMA DE OBTENÇÃO DE BIOQUEROSENE VIA CATÁLISE HETEROGÊNEA
}

\author{
F. A. F. da PONTE ${ }^{1}$, J. S. RODRIGUES ${ }^{2}$, J. Q. MALVEIRA², I. A. MACIEL ${ }^{3}$, M. C. G. \\ ALBUQUERQUE ${ }^{3}$ \\ ${ }^{1}$ Universidade Federal do Ceará, Departamento de Engenharia Química \\ ${ }^{2}$ Núcleo de Tecnologia Industrial do Ceará, Laboratório de Biocombustível \\ ${ }^{3}$ Universidade Federal do Ceará, Departamento de Engenharia Mecânica \\ E-mail: flaviodaponte@hotmail.com
}

\begin{abstract}
RESUMO: Neste trabalho foi produzido bioquerosene pela reação de esterificação (utilizando o Amberlyst ${ }^{\mathbb{R}} 15$ como catalisador heterogêneo) dos ácidos graxos livres de C6 a C16 átomos de carbono provenientes do óleo de babaçu (Orbignya speciosa) utilizando um planejamento de experimento fatorial $2^{3}$ com composto central. A análise termogravimétria foi utilizada para indicar a faixa de trabalho do catalisador e a microscopia eletronica de varredura, a morfologia. Os parâmetros de processo estudados foram a temperatura de reação entre $70{ }^{\circ} \mathrm{C}$ e $100{ }^{\circ} \mathrm{C}$, a concentração em massa de catalisador entre $5 \%$ e $10 \%$ e o tempo de reação entre 240 minutos e 480 minutos. Como parâmetro de resposta do planejamento experimental foi avaliado o teor em massa de metil ésteres. A otimização do processo foi realizada através da metodologia de superfície de resposta (MSR) e o modelo polinomial de segunda ordem. Foi observado que a variável de processo de maior influência foi a temperatura de reação, sendo que a maior conversão de ésteres foi obtida com 7,5\% de catalisador; temperatura de reação de $85^{\circ} \mathrm{C}$ e com um tempo de 360 minutos de reação. Com esses parâmetros a conversão alcançada foi de 94,7\% em biocombustível. Utilizando a MRS, com modelo polinomial de segunda ordem, as condições otimizadas obtidas para a reação de esterificação com Amberlyst ${ }^{\circledR} 15$ foram: temperatura de $90,0{ }^{\circ} \mathrm{C}$, quantidade de catalisador de 7,3 \% e tempo reacional de 338,2 minutos. O rendimento teórico da conversão previsto para os ácidos graxos livres de C6 a C16 átomos de carbono em bioquerosene foi de $95,7 \%$.
\end{abstract}

PALAVRAS-CHAVE: Bioquerosene; Acidos graxos de babaçu; Planejamento experimental; Metodologia de superfície de resposta (MSR). 


\section{INTRODUÇÃO}

A necessidade por energia proveniente dos combustíveis fósseis se tornou inerente ao ser humano, porém, esses tipos de combustíveis são prejudiciais ao meio ambiente por serem uns dos causadores do aumento dos gases de efeito estufa. Além disso, esses recursos energéticos são finitos devido a sua origem (WAKIL et al., 2015). O setor que mais cresce em escala mundial é o setor de transporte aéreo, portanto precisa de uma grande quantidade de volume de combustível (WADUD, 2015). A International Air Transport Association (IATA), em junho de 2009, estabeleceu metas de redução do uso de combustível fossíl até 2050, com o intuito de reduzir em $50 \%$ a emissão de $\mathrm{CO}_{2}$ em relação a dados de 2005 (IATA, 2009). Uma opção para atingir esse objetivo, além da melhoria na tecnologia, seria o uso de biocombustíveis.

Atualmente para a síntese dos biocombustíveis de aviação existem três principais linhas de pesquisas: querosene parafínicos sintéticos hidroprosessado por Ficher-Tropsch (SPKFT), querosene parafínicos sintéticos de ácidos graxos e ésteres hidroprocessados (SPKHEFA) e, em destaque, a de transesterificação de óleos e gorduras em biodiesel seguida da destilação e separação das frações de ésteres mais leves, em virtude de ser um método relativamente mais simples quando comparado com as demais (HONG et al., 2013).

O bioquerosene proveniente da destilação do biodiesel, que normalmente é produzido através da reação de transesterificação, é obtido através da separação em duas frações de ésteres, uma de menor e outra de maior massa molecular. A fração com menor massa molecular é o bioquerosene e a fração de maior massa molecular o biodiesel (QUERINO, 2014). Llamas et al. (2012) propuzeram misturas de ésteres metílicos de aproximadamente 8 átomos a 16 átomos de carbono na cadeia, destilado do biodiesel dos óleos de babaçu e camelina, com o querosene fóssil de aviação em teores em massa de até $10 \%$, e verificaram que dessa mistura tem-se um combustível que atende a especificação existente ASTM D1655 (2014). Ranucci et al. (2015) utilizaram em seu trabalho a mistura de ésteres metílicos destilados do biodiesel do óleo de jatropha curcas com o querosene fóssil de aviação com teores em massa de até $16 \%$ e obtiveram um produto com propriedades semelhantes a dos combustíveis fósseis disponíveis no mercado.

$\mathrm{Na}$ reação de transesterificação os triglicerídeos reagem na presença de $\mathrm{CH}_{3} \mathrm{OK}$ ou $\mathrm{CH}_{3} \mathrm{ONa}$ com um álcool de cadeia curta (RINCÓN et al., 2014). No entanto, existem alguns óleos que apresentam ácidos graxos livres (AGL) acima da quantidade adequada para este tipo de reação. Se a concentração de AGL equivalente a $\mathrm{KOH}$ for superior a $2 \mathrm{mg} / \mathrm{g}$, os sabões são formado a partir do consumo do catalisador com os AGL dificultando a purificação e o rendimento (COSTA et al., 2013; STACY et al., 2014).

Os ácidos graxos livres, extraídos dos óleos vegetais de elevado índice de acidez, podem ser uma ótima alternativa de matéria prima, para substituir os combustíveis fósseis, que são hoje amplamente utilizados, já que são de baixo custo, oriundos de diversos tipos de matérias oleaginosas, considerados renováveis, e podem ser utilizados para a produção de biocombustíveis em diversos setores (CHAI et al., 2014).

$\mathrm{O}_{2} \mathrm{SO}_{4}$ é comumente usado como um catalisador ácido homogêneo para converter AGL em biocombustível na reação de esterificação. Porém, há um grande problema na 
utilização de catalisadores homogêneos, pois exigem, após a reação, um tratamento do produto formado, gerando assim muitos resíduos prejudiciais ao meio ambiente, além de serem corrosivos aos equipamentos metálicos (CVENGROS e CVENGROSOVA, 2004). Estas desvantagens levaram à investigação do uso de resinas de troca iônicas como catalisadores sólidos para o processo de esterificação (BOZ et al., 2015).

$\mathrm{Na}$ reação de esterificação diversos parâmetros podem afetar o processo. Dentre essas variáveis, destacam-se a quantidade de catalisador, a temperatura do meio e o tempo da reação. O planejamento experimental tem como intuito a otimização e pode ser amplamente aplicado para avaliar com segurança a influência das variáveis no processo, de forma a fornecer o efeito individualmente e das possíveis interações entre as variáveis selecionadas, portanto, todas as variáveis são estudadas simultaneamente, possibilitando a melhor interpretação dos resultados. Os pesquisadores Charoenchaitrakool e Thienmethsangkoon (2011) obtiveram a otimização na produção de biocombustível a partir de óleo de fritura, no qual utilizaram um pré-tratamento com o ácido sulfúrico com finalidade de reduzir o teor de ácidos graxos livres (AGL) do meio para um valor de $0,5 \%$. A melhor condição encontrada foi com $0,7 \%$ de ácido, razão molar metanol/óleo de $6: 1$ e em temperatura de $51{ }^{\circ} \mathrm{C}$ por 60 minutos de reação. Felizardo et al. (2006) utilizaram o planejamento experimental para investigar quais seriam as melhores condições de produção de biodiesel com o óleo de fritura e o uso de um catalisador homogêneo básico $(\mathrm{NaOH})$ através da observação do efeito negativo no rendimento da reação com o aumento da acidez. Liu et al. (2014) investigaram as condições ótimas do processo de esterificação dos ácidos graxos livres do óleo, utilizando o etanol como álcool esterificante na presença de um catalisador sólido (PA/NaY). A melhor condição operacional atingiu conversão de $79,5 \%$ e foi com a razão molar álcool:ácido oléico de 7:1, com 1,7 g de catalisador a $105{ }^{\circ} \mathrm{C}$ durante 7 horas. Tiwari et al. (2007) reportaram com a utilização do planejamento experimental a viabilidade do processo de esterificação dos ácidos graxos livres do óleo de Jatropha curcas, que reduziu o teor mássico de $14 \%$ para o valor menor do que $1 \%$. O ótimo do processo foi com $1,4 \%$ de catalisados, razão volumétrica (metanol e óleo) de 0,28, tempo reacional de 88 minutos a uma temperatura de $60{ }^{\circ} \mathrm{C}$. Com esse procedimento foi obtido um rendimento médio em biocombustível por volta de $99 \%$. Gan et al. (2012) estudaram a influência de três diferentes resinas de troca iônicas, como catalisadores na otimização da reação de esterificação do óleo de fritura com elevado teor de AGL. Observaram que das três resinas, a Amberlyst 15 foi a que obteve a maior conversão, com os seguintes parâmetros: $4 \%$ em peso de catalisador, temperatura de $65{ }^{\circ} \mathrm{C}$ em uma razão molar de óleo/metanol de 1:15. Sob estas condições os pesquisadores obtiveram uma conversão máxima de AGL em ésteres de 60,2 \%.

Para melhorar a conversão dos AGL em bioquerosene, a metodologia de superfície de resposta (MSR) pode ser utilizada para otimizar os parâmetros reacionais. A MSR é vantajosa, pois permite obter grandes quantidades de informações a partir de experimentos reduzidos, além de fornecer a possibilidade de observar os efeitos das variáveis individuais e suas combinações de interações sobre a resposta (KUMAR et al., 2014; LIU et al., 2014).

Há uma escassez de literatura quanto a aplicação de processos de otimização com o uso das resinas de troca iônicas comerciais, como catalisadores para a obtenção de biocombustível. Um estudo global, que avalie as principais variáveis na reação de esterificação para a produção do bioquerosene via catálise heterogênea, mostra-se 
interessante e pode vir a contribuir com dados fundamentais importantes e um bioproduto possível de ser utilizado em mistura ao querosene fóssil de aviação.

O objetivo do presente trabalho foi de otimizar as variáveis de produção do bioquerosene via catálise heterogênea usando o catalisador Amberlyst ${ }^{\circledR} 15$, com o auxílio do planejamento experimental fatorial e metodologia de superfície de resposta, a fim de alcançar a máxima eficiência na reação de esterificação dos ácidos graxos livres (C6 a C16), obtidos na destilação do óleo de babaçu. A concentração do catalisador, temperatura e tempo reacional foram os parâmetros estudados neste trabalho.

\section{MATERIAIS E MÉTODOS}

Foram utilizados ácidos graxos de 6 átomos a 16 átomos de carbonos, obtidos na destilação do óleo de babaçu; álcool metílico (99,9 \%) adquirido da Dinâmica ${ }^{\circledR}$ e catalisador Amberlyst $^{\circledR} 15$ adquirido SigmaAldrich ${ }^{\circledR}$.

\subsection{Obtenção dos ácidos graxos}

A separação dos ácidos graxos livres (AGL) do óleo de babaçu (Orbignya speciosa) na faixa de 6 átomos a 16 átomos de carbonos foi realizada através de um sistema de destilação fracionada à vácuo, com refrigeração e acoplado a duas bombas em série, no qual os ácidos graxos foram destilados na faixa de temperatura da manta aquecedora de $140{ }^{\circ} \mathrm{C}$ a $180{ }^{\circ} \mathrm{C}$ em pressão reduzida de 0,01 bar $(1,33 \mathrm{Kpa})$ como mostra a Figura 1.

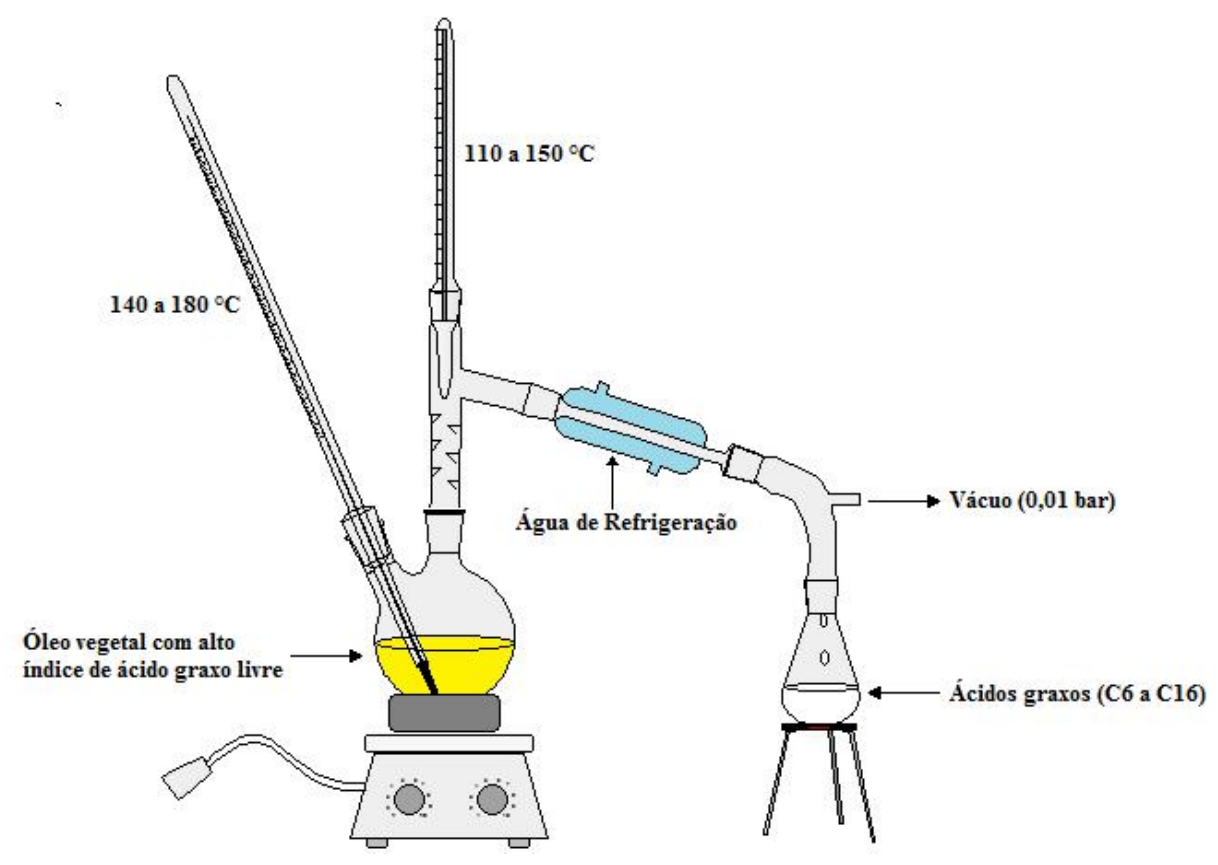

Figura 1 - Sistema representativo da destilação fracionada a vácuo do óleo de babaçu. 


\subsection{Análise de composição}

As composições, tanto do óleo de babaçu como dos ácidos graxos destilados, foram analisadas em um CG/MS QP2010 (Shimadzu, Japão) equipado com uma coluna capilar Rtx$5 \mathrm{MS}$ de $30 \mathrm{~m}$ de comprimento, $0,25 \mathrm{~mm}$ de diâmetro e espessura de $0,25 \mu \mathrm{m}$. O gás de arraste utilizado foi o hélio, a uma taxa de fluxo de $5,0 \mathrm{~mL} \mathrm{~min}{ }^{-1}$. A taxa de fluxo de purga foi de 3,0 mL min-1 e a velocidade linear de $36,3 \mathrm{~cm} \mathrm{~s}^{-1}$. A temperatura foi programada como se segue: iniciou-se a $50{ }^{\circ} \mathrm{C}$, seguido de um taxa gradual de $15{ }^{\circ} \mathrm{C} \mathrm{min}{ }^{-1}$ até atingir $270{ }^{\circ} \mathrm{C}$ esta temperatura mantendo por 20 minutos, em seguida deu-se seguência a rampa de aquecimento até $300{ }^{\circ} \mathrm{C}$ com uma taxa de $10{ }^{\circ} \mathrm{C} \mathrm{min}{ }^{-1}$. A temperatura do injetor foi de $250{ }^{\circ} \mathrm{C}$ e interface de fonte de íons de $260^{\circ} \mathrm{C}$.

\subsection{Análise do catalisador}

A análise termogravimétrica (TGA) e a derivada da TGA (DTG) do catalisador heterogêneo, Amberlyst ${ }^{\circledR}$ 15, foi realizada com o propósito de verificar em qual temperatura ocorreria a completa decomposição da resina polimérica, pois no planejamento experimental o catalisador deve ficar em uma faixa de trabalho confiável, garantindo assim a atividade do catalisador. O equipamento utilizado nesta análise foi DTG-TG $60 \mathrm{H}$ da Shimadzu. A análise térmica foi realizada sob fluxo de ar sintético $\left(40 \mathrm{~mL} \mathrm{~min}^{-1}\right)$ à temperatura de $24{ }^{\circ} \mathrm{C}$ até 1000 ${ }^{\circ} \mathrm{C}$ com uma taxa de aquecimento de $10{ }^{\circ} \mathrm{C} \mathrm{min}^{-1}$. A massa utilizada no porta amostra de alumínio foi de $10,443 \mathrm{mg}$. A morfologia da superfície e a composição química da Amberlyst ${ }^{\circledR} 15$ foram documentadas usando um microscópio eletrônico de varredura (MEV) FEG quanta 450 ambiental com EDS/EBSD, com estágio de resfriamento e aquecimento.

\subsection{Planejamento Composto Central}

O planejamento composto central (PCC) com três fatores foi aplicado para analisar a influência das condições operacionais na produção de biocombustível via esterificação heterogênea. Como apresentado na Tabela 1, as variáveis de estudo foram: concentração de catalisador $\left(\mathrm{X}_{1}\right)$, temperatura da reação $\left(\mathrm{X}_{2}\right)$ e o tempo reacional $\left(\mathrm{X}_{3}\right)$. Como parâmetro de resposta foi avaliado o teor em massa de ésteres metílicos. O planejamento resultou em um total de 17 experimentos, sendo 6 experimentos axiais, 8 experimentos cúbicos e 3 experimentos centrais. Para minimizar os erros sistemáticos as reações foram realizadas de forma aleatória. A interpretação dos dados foi realizada pela análise de variância do modelo matemático aplicado na metodologia de superfície de resposta (MSR). Para o tratamento dos dados e obtenção dos gráficos estatísticos foi utilizado o programa Statistica ${ }^{\circledR}$ 7.0.

A aplicação do planejamento e a determinação das condições ótimas experimentais teve o objetivo de possibitar a formação de um elevado teor de metil ésteres na reação de esterificação. Para isso, seguiu o esquema da Figura 2. As variáveis foram codificadas nos níveis inferiores e superiores de $-1 \mathrm{a}+1$, respectivamente, para pontos experimentais cúbicos e 0 para pontos experimentais centrais. Já para os experimentos axiais os valores para os níveis inferiores e superiores foram de $-1,7$ e $+1,7$, respectivamente, como apresentado na Tabela 1. 


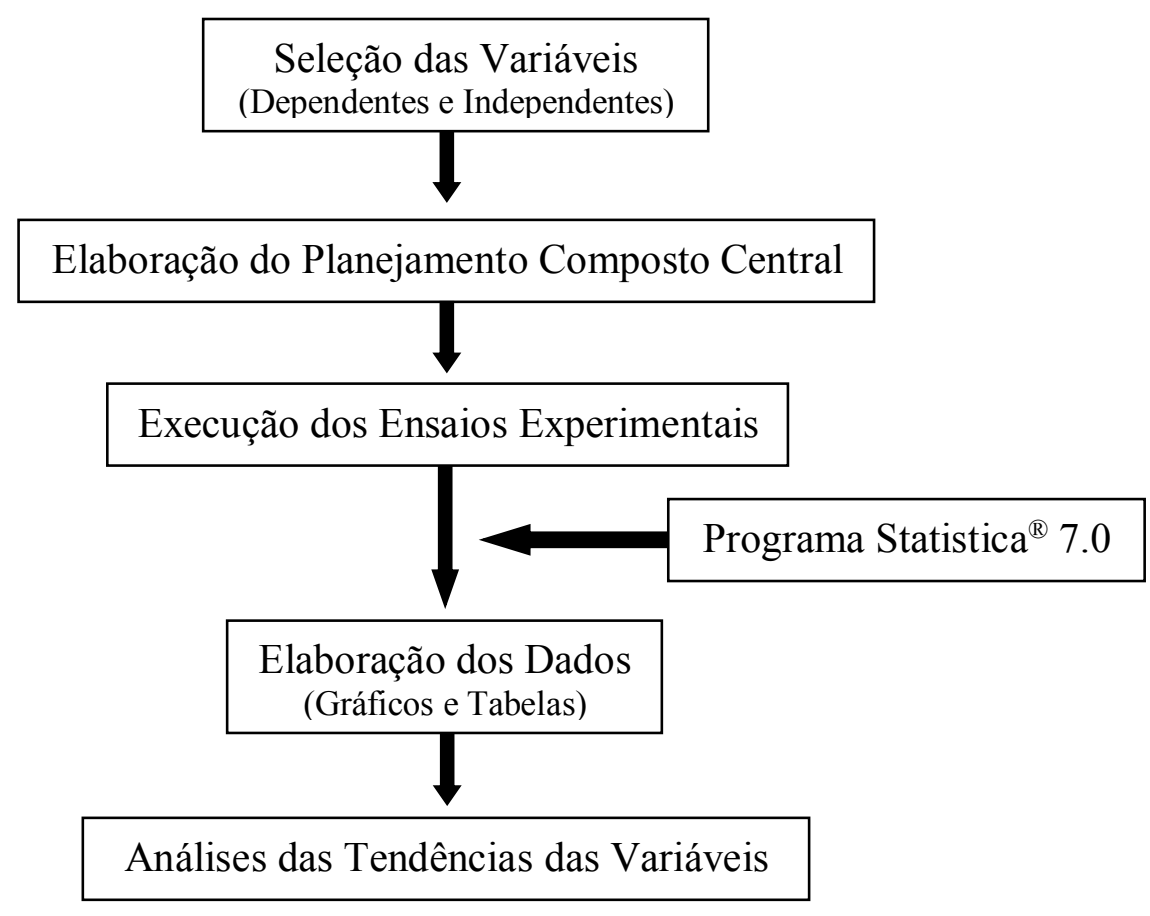

Figura 2 - Esquema utilizado para a triagem das variáveis da reação.

Tabela 1 - Planejamento composto central realizado.

\begin{tabular}{lcccccc}
\hline \multirow{2}{*}{ Variáveis } & \multirow{2}{*}{ Símbolo } & \multicolumn{5}{c}{ Nível } \\
\cline { 3 - 7 } & & $\mathbf{- 1 , 7}$ & $\mathbf{- 1}$ & $\mathbf{0}$ & $+\mathbf{1}$ & $+\mathbf{1 , 7}$ \\
\hline Catalisador (\%peso) & $\mathrm{X}_{1}$ & 3,1 & 5 & 7,5 & 10 & 11,8 \\
Temperatura $\left({ }^{\circ} \mathbf{C}\right)$ & $\mathrm{X}_{2}$ & 59,4 & 70 & 85 & 100 & 110,5 \\
Tempo (minutos) & $\mathrm{X}_{3}$ & 156 & 240 & 360 & 480 & 564 \\
\hline
\end{tabular}

\subsection{Reação de esterificação}

As sínteses de bioquerosene foram realizadas em reator de aço inox $(50 \mathrm{~mL})$ totalmente fechado acoplado a uma chapa aquecedora da marca IKA, provido de um manômetro, agitação magnética constante de $1.100 \mathrm{rpm}$ e controlador de temperatura. A reação do AGL com o álcool é estequiometricamente de 1:1, como mostra a Figura 3. Para o estudo, a relação molar entre os ácidos graxos livres destilados do óleo de babaçu e o álcool (metanol) foi fixada em 1:6 para todas as reações, de acordo com resultados de testes preliminares nos quais foram observados que um excesso de álcool nesta proporção favorece o deslocamento da reação para a formação dos produtos. A temperatura, a concentração de catalisador e o tempo reacional foram variados para cada reação de acordo com o PCC. Antes de cada reação do planejamento, o catalisador foi mantido em temperatura constante de $60{ }^{\circ} \mathrm{C}$, na estufa por 24 horas com o intuito de remover a umidade, e em seguida pesados de acordo com o planejamento experimental proposto. Após a reação de esterificação, o catalisador foi separado por filtração e a fase líquida foi levada à um funil de decantação com o intuito de 
separar o álcool excedente e a água formada do produto final (biocombustível). O processo de produção do bioquerosene, utilizado neste trabalho, encontra-se descritos pelo fluxograma apresentado na Figura 4.

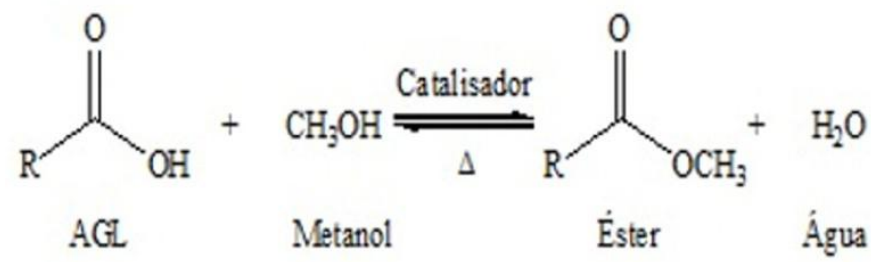

Figura 3 - Reação de esterificação dos ácidos graxos livres.

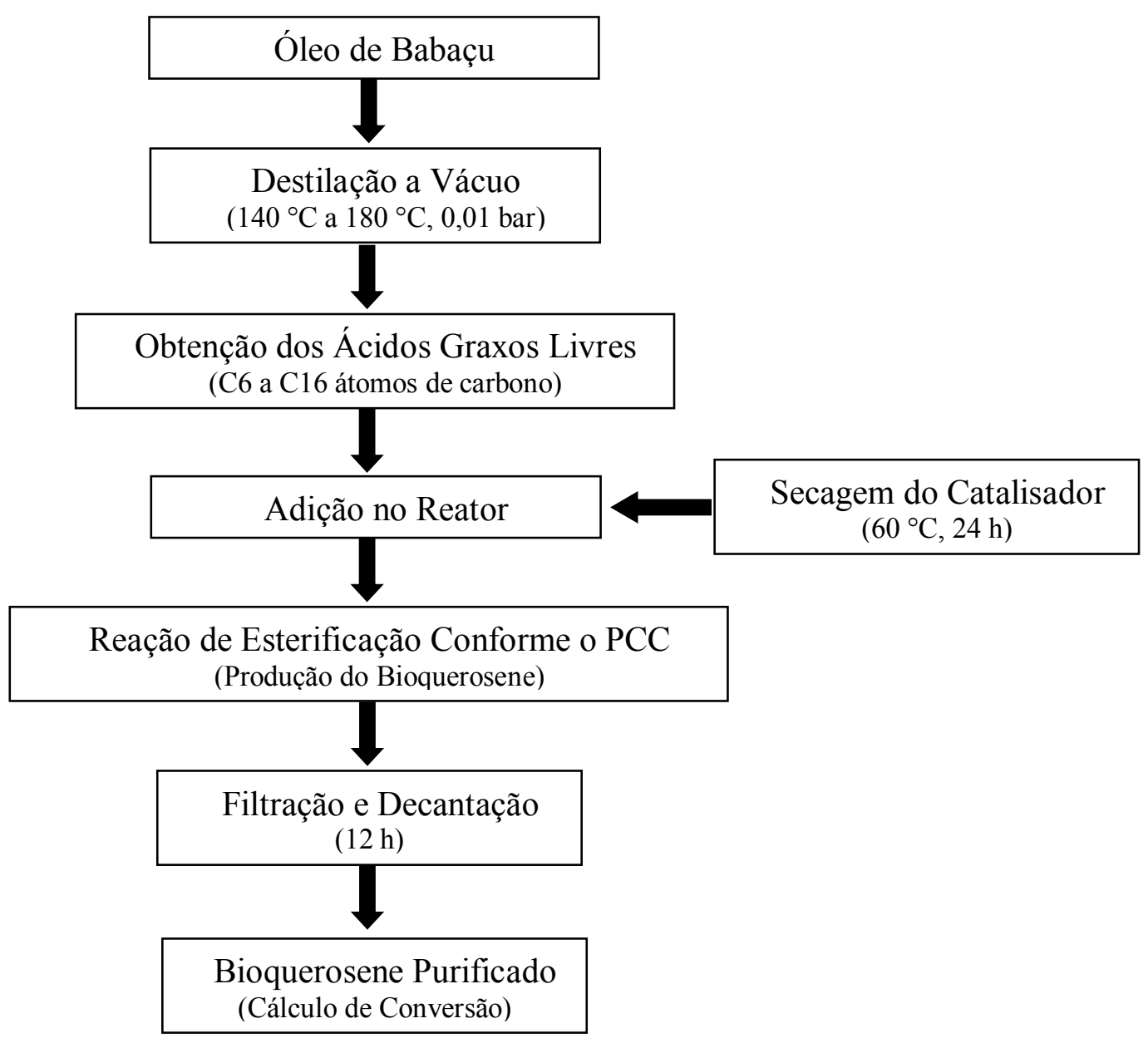

Figura 4 - Fluxograma do processo de produção do bioquerosene.

\subsection{Conversão em bioéster}

A conversão do bioproduto formado foi determinada a partir do índice de acidez, antes e após a reação de esterificação. Para determinação do índice de acidez, 2 g da amostra foram 
dissolvidas em $25 \mathrm{~mL}$ de uma mistura de éter e álcool etílico $2: 1 \mathrm{v} / \mathrm{v}$ (previamente neutra com $\mathrm{NaOH} 0,1 \mathrm{~mol} \mathrm{~L}^{-1}$ na presença do indicador fenolftaleína). Em seguida a mistura foi titulada com a solução de hidróxido de sódio $(\mathrm{NaOH}) 0,1 \mathrm{~mol} \mathrm{~L}^{-1}$ até o aparecimento da coloração rósea, a qual permaneceu por 30 segundos. O procedimento foi realizado conforme o descrito no manual de métodos do Instituto Adolfo Lutz (2005).

O cálculo do teor de ésteres metílicos, resposta do planejamento, foi feito utilizando o índice de acidez inicial da amostra e o índice de acidez do produto da reação de esterificação, de acordo com a Equação 1.

$$
B E_{(\%)}=\frac{\left(I_{A C}-I_{B}\right) \times 100}{I_{A C}}
$$

Onde $\mathrm{BE}_{(\%)}$ representa o teor em massa de ésteres metílicos (\%) (bioquuerosene); $\mathrm{I}_{\mathrm{AC}}$ é $\mathrm{o}$ índice de acidez do material de partida e o $\mathrm{I}_{\mathrm{B}}$ é o índice de acidez do biocombustível.

\section{RESULTADOS E DISCUSSÃO}

\subsection{Análise da composição}

A Tabela 2 apresenta a composição química do óleo de babaçu e do ácido graxo obtidos na destilação deste óleo.

Tabela 2 - Composição química do óleo de babaçu e do ácido graxos obtidos na destilação.

\begin{tabular}{lcc}
\hline Composição dos ácidos graxos & Óleo de babaçu & $\begin{array}{c}\text { Ácidos graxos livres } \\
\text { destilados }\end{array}$ \\
\hline C6:0 Capróico & $0,2 \%$ & $4,1 \%$ \\
C8:0 Caprílico & $13,7 \%$ & $26,0 \%$ \\
C10:0 Cáprico & $14,7 \%$ & $18,0 \%$ \\
C12:0 Láurico & $57,5 \%$ & $33,4 \%$ \\
C14:0 Mirístico & $9,6 \%$ & $12,6 \%$ \\
C16:0 Palmítico & $2,3 \%$ & $5,9 \%$ \\
C18:0 Esteárico & $0,4 \%$ & $0 \%$ \\
C18:1 Oléico & $1,4 \%$ & $0 \%$ \\
C18:2 Linoléico & $0,2 \%$ & $0 \%$ \\
\hline
\end{tabular}

Com a utilização da técnica de destilação à pressão reduzida foi possível a obtenção dos ácidos graxos livres na faixa de 6 átomos a 16 átomos de carbono. De acordo com a Tabela 2 a composição química deste material é constituída principalmente de quatro ácidos graxos: ácido caprílico ( 8 átomos de carbono) $26 \%$, ácido cáprico (10 átomos de carbono) 18 $\%$, ácido mirístico (14 átomos de carbono) 12,6\% e uma concentração majoritária do ácido láurico (12 átomos de carbono) 33,4\%, presentes apenas ácidos graxos saturados. Neste caso, o material obtido pode ser um precursor na produção do bioquerosene, pois apresenta 
maiores composições de ácidos graxos na faixa de 6 átomos a 16 átomos de carbono que corresponde a faixa de destilação do querosene fóssil, que é de 8 átomos a 16 átomos de carbono.

\subsection{Análise do catalisador}

Análise Termogravimétrica: Na Figura 5 está apresentada a curva termogravimétrica da Amberlyst ${ }^{\circledR} 15$.

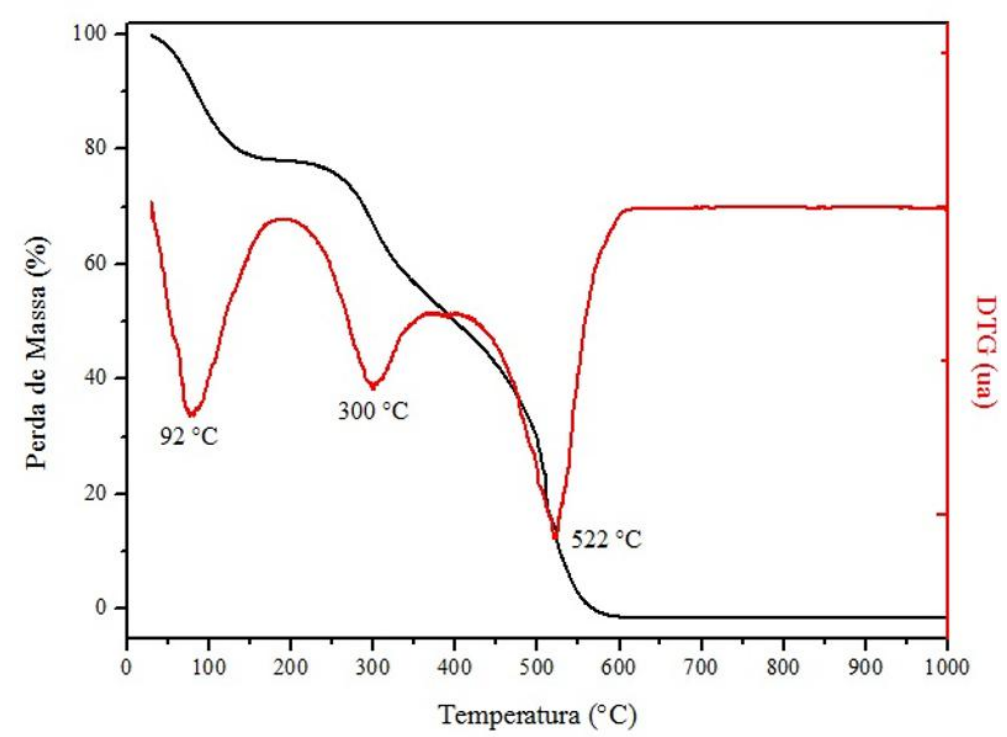

Figura 5 - Análise termogravimétrica da Amberlyst ${ }^{\circledR} 15$.

Conforme as curvas TGA e DTG mostradas na Figura 5, observou-se que a perda de massa foi composta por três eventos. Estes eventos foram mais evidenciados com a curva de DTG.

$\mathrm{O}$ primeiro evento começou, aproximadamente, em $60{ }^{\circ} \mathrm{C}$ e foi até $150{ }^{\circ} \mathrm{C}$, sendo caracterizado através da derivada primeira da curva TGA em torno de $92{ }^{\circ} \mathrm{C}$, indicando presença de água adsorvida na amostra. Li et al. (2009), também fizeram esta observação ao estudarem a mesma faixa de temperatura. Fan et al. (2014) em seus estudos com Amberlyst ${ }^{\circledR}$ 15 reciclado de reação da celulose em acetato de celulose, verificaram que a massa do catalisador reutilizado foi ligeiramente menor do que a do catalisador novo, após as reações. Estas perdas de massa foram atribuídas, principalmente, a perda de água física molecularmente adsorvidas.

O segundo evento que foi de $200{ }^{\circ} \mathrm{C}$ a $400{ }^{\circ} \mathrm{C}$, com pico máximo em $300{ }^{\circ} \mathrm{C}$ na DTG, esta faixa pode ser atribuída à despolimerização das cadeias carbônica do polímero e a decomposição dos grupos sulfônicos ácidos $\left(-\mathrm{SO}_{3} \mathrm{H}\right)$ do catalisador sólido. Nesta faixa de temperatura, os pesquisadores Ciopec et al. (2013) relataram que o sítio ativo é totalmente afetado pela degradação, portanto perdendo seu caráter de catalisador.

$\mathrm{O}$ terceiro evento que começou, aproximadamente, em $450{ }^{\circ} \mathrm{C}$ e terminou em $600{ }^{\circ} \mathrm{C}$, com pico máximo em $510{ }^{\circ} \mathrm{C}$ na DTG, esta faixa foi atribuída a degradação completa do catalisador sólido, visto que depois disso não foi registrado mais nenhum evento. De acordo com a curva, o intervalo de temperatura estabelecido no planejamento experimental ficou abaixo da temperatura de degradação do catalisador, logo dentro da faixa de trabalho da Amberlyst ${ }^{\circledR} 15$. 
Microscopia de varredura: Como pode ser visto na Figura 6, a Amberlyst ${ }^{\circledR} 15$ apresenta-se com aspecto esférico de diâmetro de $613 \mu \mathrm{m}$ e superfície lisa. A análise de Espectroscopia de energia dispersiva (EDS) mostrou que a composição química do catalisador é constituída de carbono, oxigênio, enxofre e hidrogênio.

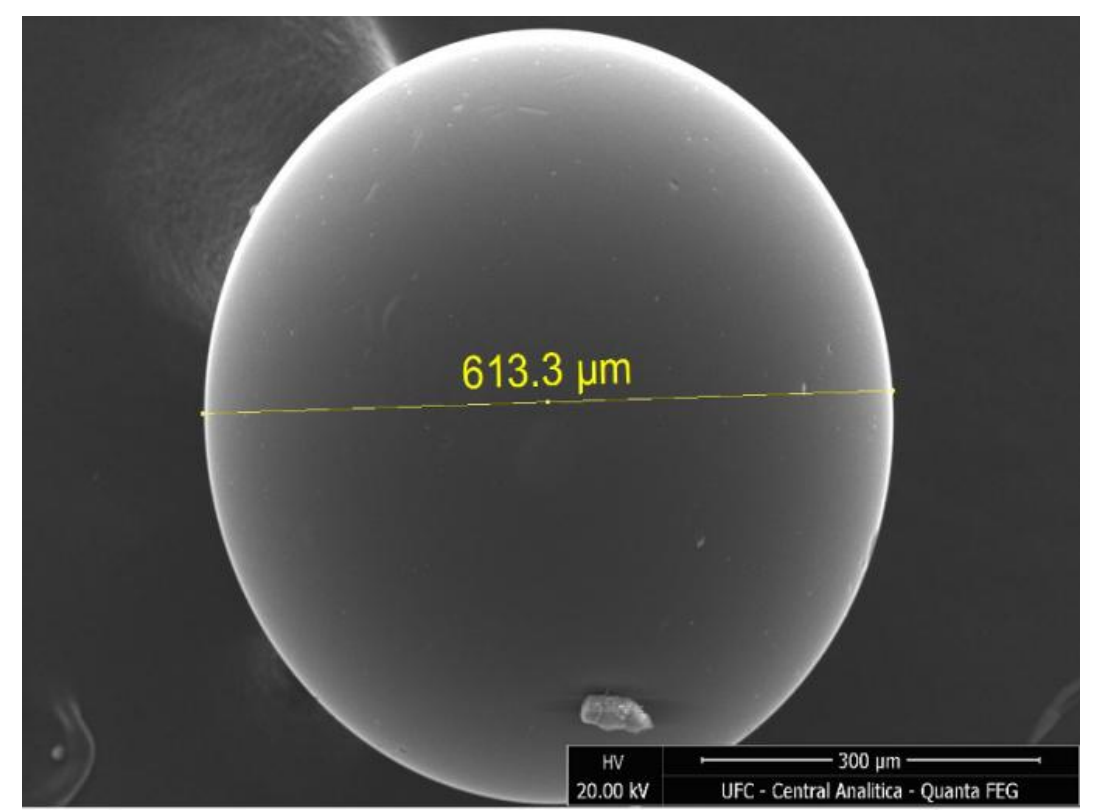

Figura 6 - Imagem do microscópio eletrônico de varredura (MEV).

\subsection{Planejamento experimental}

Planejamento composto central (PCC): A matriz de planejamento, com os resultados obtidos em teor de massa de metil ésteres estão apresentados na Tabela 3.

O planejamento descrito na Tabela 3 para a produção do bioquerosene a partir dos ácidos graxos de 6 átomos a 16 átomos de carbonos, obtidos na destilação do óleo de babaçu aplicado neste trabalho foi considerado satisfatório, por apresentar conversões em torno de $94 \%$ em teor de ésteres formados. Pois, em estudos anteriores a este trabalho, foi obtido um bioquerosene com $92 \%$ em teor em massa de ésteres metílicos, e este bioproduto possuia as características físico-químicas semelhantes ao querosene fóssil de aviação (PONTE et al., 2015). A melhor conversão em teor de ésteres metílicos obtida no planejamento experimental foi encontrada em uma das réplicas do ponto central, que foi nas condições: 7,5\% em peso de catalisador; temperatura de reação de $85^{\circ} \mathrm{C}$ e 360 minutos de reação. 
Tabela 3 - Matriz do Planejamento composto central (PCC).

\begin{tabular}{ccccc}
\hline \multirow{2}{*}{ Reações } & \multicolumn{3}{c}{ Variáveis dependentes } & \multirow{2}{*}{ Teor em massa de metil ésteres (\%) } \\
\cline { 2 - 4 } & $\mathbf{X}_{\mathbf{1}}$ & $\mathbf{X}_{\mathbf{2}}$ & $\mathbf{X}_{\mathbf{3}}$ & \\
\hline 1 & -1 & -1 & -1 & 68,55 \\
2 & -1 & -1 & +1 & 81,88 \\
3 & -1 & +1 & -1 & 92,36 \\
4 & -1 & +1 & +1 & 92,59 \\
5 & +1 & -1 & -1 & 80,86 \\
6 & +1 & -1 & +1 & 82,97 \\
7 & +1 & +1 & -1 & 93,70 \\
8 & +1 & +1 & +1 & 89,75 \\
9 & $-1,7$ & 0 & 0 & 85,34 \\
10 & $+1,7$ & 0 & 0 & 87,69 \\
11 & 0 & $-1,7$ & 0 & 70,45 \\
12 & 0 & $+1,7$ & 0 & 91,42 \\
13 & 0 & 0 & $-1,7$ & 82,80 \\
14 & 0 & 0 & $+1,7$ & 85,17 \\
15 & 0 & 0 & 0 & 93,41 \\
16 & 0 & 0 & 0 & 90,72 \\
17 & 0 & 0 & 0 & 94,70 \\
\hline
\end{tabular}

Análise de variância: A partir de uma avaliação estatística dos resultados dos 17 experimentos da Tabela 3, foi realizada a análise de variância (ANOVA), que se encontra na Tabela 4. Por meio do valor de $\mathrm{F}$ foi possível avaliar a significância estatística do modelo utilizado em função do erro experimental indeterminado, de acordo com a Equação 2.

$F_{\text {calc }}=\frac{M S Q_{\text {regressão }}}{M S Q_{\text {residuos }}}$

Tabela 4 - Análise de variância (ANOVA) para a avaliação do modelo de produção do bioquerosene.

\begin{tabular}{|c|c|c|c|c|c|c|}
\hline Fonte de variação & SQ & GL & MSQ & $\mathbf{F}_{\text {calc }}$ & $F_{\operatorname{tab}(0,05 ; 9 ; 7)}$ & $\mathbf{P}_{\text {calc }}$ \\
\hline Regressão & 1008,39 & 9 & 112,04 & 24,20 & 3,68 & 0,000182 \\
\hline Resíduos & 32,39 & 7 & 4,63 & & & \\
\hline Falta de ajuste & 24,14 & 5 & 4,83 & & & \\
\hline Erro puro & 8,25 & 2 & 4,12 & & & \\
\hline Total & 976 & 16 & 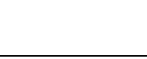 & 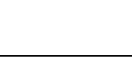 & 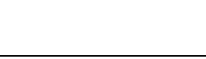 & 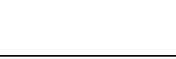 \\
\hline
\end{tabular}

A maneira utilizada para avaliar a correlação existente entre fatores e resposta, qualidade do ajuste, foi através do valor de F na Regressão. Quando o $F_{\text {calc }}$ é maior do que o 
$\mathrm{F}_{\text {tab }}\left(\mathrm{F}_{\text {calculado }}>\mathrm{F}_{\text {tabelado }}\right)$, o modelo é satisfatório. Analisando os resultados obtidos na Tabela 4, foi verificado que o valor do $F_{\text {calc }}(24,20)$ foi 6 vezes maior do que o valor tabelado de $\mathrm{F}_{\operatorname{tab}(0,05 ; 9 ; 7)}(3,68)$. Analisando agora o valor de $\mathrm{P}$ foi observado que este parâmetro foi menor do que 0,05 , o nível de significância requerido no modelo, mostrando que o modelo é preditivo no nível de confiança de $95 \%$. Portanto, foi altamente significativo o modelo proposto $\left(\mathrm{P}_{\text {calc }}<0,05\right.$ e $\left.\mathrm{F}_{\text {calc }}>\mathrm{F}_{\text {tab }}\right)$.

Os gráficos mostrados na Figura 7 foram usados para analisar a qualidade do ajuste do modelo proposto. Na Figura 7a, observa-se que os valores previstos versus os valores observados, apresenta um bom ajuste do modelo proposto, podendo-se observar que os resíduos são pequenos, justificado pelo coeficiente de determinação $\left(\mathrm{R}^{2}\right)$, igual a 0,9660 , indicando que $96,6 \%$ da variação na produção do bioquerosene pode ser explicado. Outra forma de análisar a qualidade do modelo, encontra-se na Figura $7 \mathrm{~b}$, o gráfico de probabilidade normal dos resíduos, que indicou que os pontos experimentais estavam próximos da linha contínua, portanto, pode-se dizer que os resíduos seguem uma distribuição normal e o modelo proposto é satisfatório.

(a)

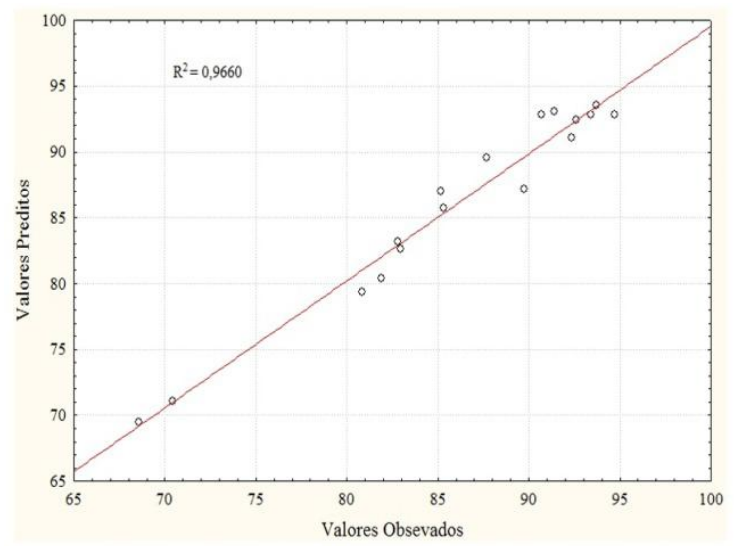

(b)

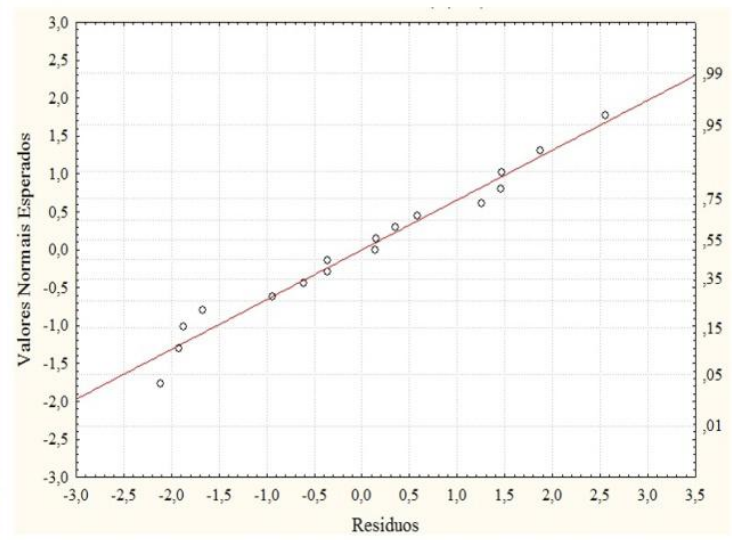

Figura 7 - Gráficos: a) valores previtos versus observados e b) probabilidade normal dos resíduos.

Metodologia de superfície de resposta (MSR): O Método de superfície de resposta é uma técnica estatística muito útil para a modelagem e análise de problemas nos quais as respostas são influenciadas por diversas variáveis com o objetivo de otimizar os dados. A partir do modelo polinomial de segunda ordem que foi analisado através do MSR, foi possível obter a Equação 3, que descreveu o comportamento da resposta, subsequentemente, elucidando as interações entre os fatores estudados com intervalo de confiança de $95 \%$.

$f(X)=92,8290+1,1607 X_{1}+6,5467 X_{2}+1,1429 X_{3}-1,8287 X_{1}^{2}-3,8015 X_{2}^{2}-2,6736 X_{3}^{2}-$ $1,8625 X_{1} X_{2}-1,9250 X_{1} X_{3}-2,3950 X_{2} X_{3}$

O diagrama de pareto, mostrado na Figura 8, ilutra a influência das variáveis (lineares e quadráticas) e suas interações na conversão em bioquerosene. A magnitude de cada efeito é 
indicado atráves da barra horizontal que excedem a linha vertical tracejada $(\mathrm{P}=0,05)$ correspondendo ao nivel de $95 \%$ de confiança, os quais são mais significantes na produção do bioquerosene.

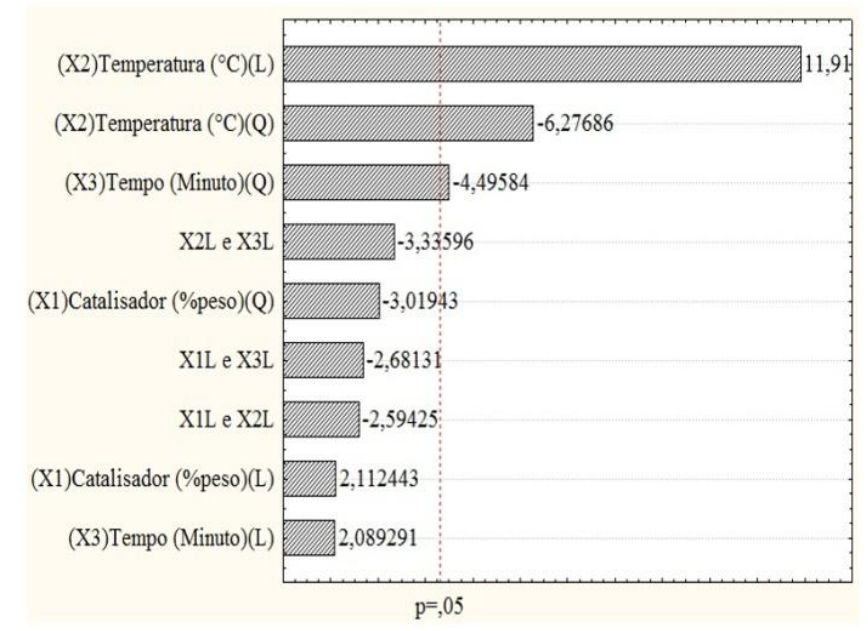

Figura 8 - Diagrama de Pareto: análise dos efeitos das variáveis na produção do bioquerosene. (L) efeitos lineares e (Q) efeitos quadráticos.

De acordo com o observado na Figura 8, entre as variáveis estudadas, as que apresentaram maior efeito significativo em um intervalo de confiança de $95 \%$ foram: a temperatura, com efeito linear (L) e quadrático (Q); e o tempo reacional, com o efeito quadrático (Q). A explicação para que a variável de maior significância seja a temperatura, deve-se pelo fato do aumenta da energia cinética dos materiais envolvidos, fazendo com que o aumente da colisão entre as moléculas com a energia de ativação necessária aconteça com maior facilidade. Em relação à variável tempo, relaciona-se ao equilíbrio da reação.

As variáveis não podem ser analisadas isoladamente e devem ser consideradas as interações entre elas. Os efeitos por interação dos fatores $\left(\mathrm{X}_{1}\right)$ e $\left(\mathrm{X}_{2}\right),\left(\mathrm{X}_{1}\right)$ e $\left(\mathrm{X}_{3}\right),\left(\mathrm{X}_{2}\right)$ e $\left(\mathrm{X}_{3}\right)$ possuem valores negativos, o que indica que o resultado é a diminuição do teor de massa do metil ésteres na reação de esterificação. Portanto, para aumentar o teor de massa de ésteres metílicos, deve-se inverter a tendência das variáveis individuais de menor significância que a interação das variáveis. Além disso, o efeito da interação dos fatores $\left(\mathrm{X}_{1}\right)$ e $\left(\mathrm{X}_{3}\right)$ foi maior do que os de suas variáveis isoladas na reação de esterificação, porém não foi significativo (confiança do modelo foi menor que $95 \%$ ). Analisando o diagrama de Pareto (Figura 8) foi observado que as interações $\left(\mathrm{X}_{1}\right)$ e $\left(\mathrm{X}_{2}\right),\left(\mathrm{X}_{2}\right)$ e $\left(\mathrm{X}_{3}\right)$ também não são significativas (confiança do modelo foi menor que $95 \%$ ), por isso foi utilizado a MSR para analisar a interação das variáveis.

Os efeitos entre as variáveis na produção do biocombustível podem ser melhor observados na Figura 9, através da superfície de resposta, representando a resposta (teor em massa de ésteres metílicos) em função das interação das variáveis, dentro do alcance experimental considerado. 
(a)

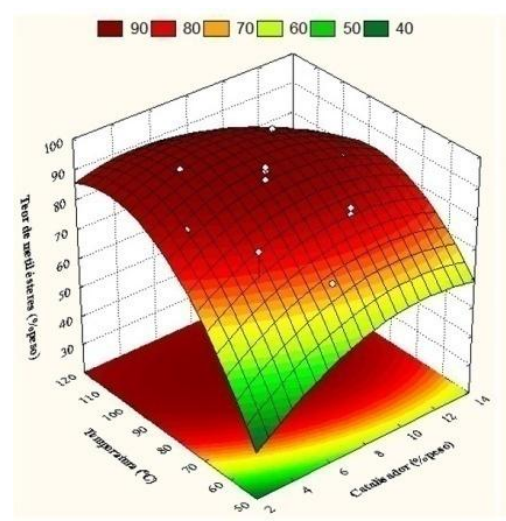

(b)

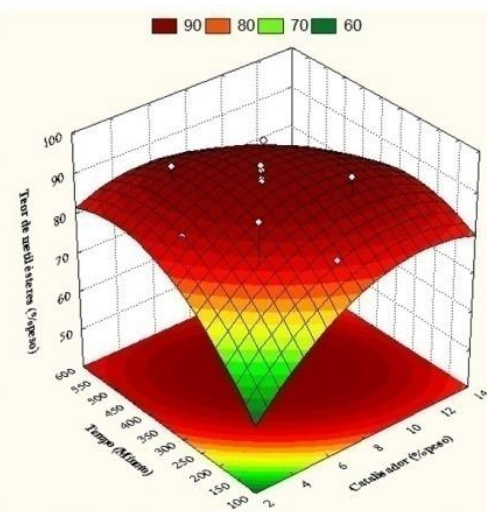

(c)

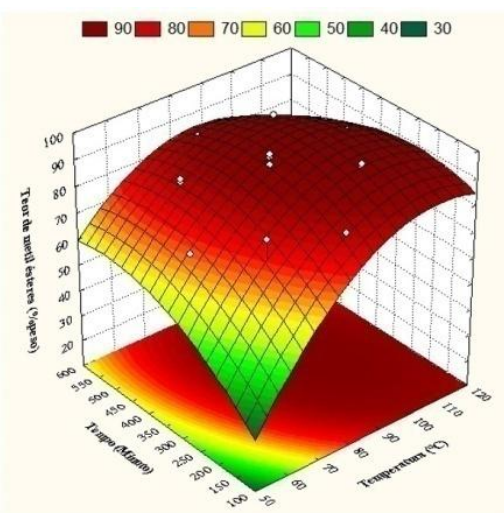

Figura 9 - Gráfico de superfície de resposta em função das interações: (a) temperatura versus catalisador; (b) tempo versus catalisador e (c) tempo versus temperatura.

Analisando os gráficos da Figura 9, observa-se que há um ponto ótimo com a utilização destas variáveis. Na Figura 9a, pode ser observado o efeito da temperatura e a quantidade de catalisador na conversão de ácidos graxos de 6 átomos a 16 átomos de carbono em bioquerosene, verifica-se que a variável temperatura influencia na produção do bioquerosene. A maior concentração em bioquerosene pode ser alcançada com temperaturas acima de $80{ }^{\circ} \mathrm{C}$. Já a quantidade de catalisador tem pouca representabilidade na reação. Outra forma de averiguar a pouca influência da quantidade da Amberlyst ${ }^{\circledR} 15$ na reação de produção do bioquerosene é analisando o gráfico da Figura 9b, pois a maior conversão (90 \%), encontra-se no centro do gráfico de superfície de resposta e as linhas de contorno são circulares, portanto, de acordo com as observações de Dong et al. (2016), linhas de contorno circulares ocorrem quando a interação entre os dois fatores são desprezável. Os pesquisadores De Lima et al. (2013) revelaram que a alta concentração do catalisador não são garantias de rendimento elevados, o que explica o ótimo ser no centro do gráfico de superfície de resposta. Para avaliar a importância do catalisador na reação foi realizado um teste nas condições: temperatura de $100{ }^{\circ} \mathrm{C}$ e 240 minutos de tempo reacional, mas na ausência do catalisador, como resultado foi obtido $21,16 \pm 0,20 \%$ de conversão. Portanto, apresença do catalisador é necessária para a produção do bioquerosene nas condições estudadas. Na Figura 9c, observase que com o aumento no tempo e na temperatura de reação, a conversão em ésteres aumentam, atingindo seu valor máximo, aproximadamente, no tempo entre 300 minutos a 400 minutos e na temperatura entre $90{ }^{\circ} \mathrm{C}$ a $110^{\circ} \mathrm{C}$. De acordo com Avramovic et at. (2015) a alta inclinação do gráfico de superfície de resposta indica grande influência de ambos os fatores no processo reacional. Um aumento no valor das correspondentes variáveis, acima deste valores citados, promove um decréscimo na conversão em ésteres. Portanto, as condições ótimas, utilizando estas variáveis de estudos, para se alcançar máximas conversões em ésteres seriam: $7,34 \%$ em massa de catalisador, $99{ }^{\circ} \mathrm{C}$ de temperatura reacional e 338 minutos de reação.

Os resultados evidenciaram que uma das principais vantagens da utilização do planejamento experimental foi a possibilidade de avaliar o sistema de maneira multivariada, devido a isto foi possível obter dados confiavéis para o sistema em estudo. 


\section{CONCLUSÃO}

A análise termogravimétrica do catalisador Amberlyst ${ }^{\circledR} 15$ mostrou que a faixa de temperatura reacional proposta no planejamento foi adequada, pois foi utilizado o catalisador dentro de sua faixa de trabalho, no qual sua estrutura química encontra-se em perfeito estado. A imagem de MEV indicou que a catálise ocorre na superfície da resina de troca iônica $\left(\right.$ Amberlyst ${ }^{\circledR}$ 15). O planejamento experimental permitiu a triagem e a determinação dos valores das variáveis que conduzem ao maior teor de ésteres metílicos. Os valores otimizados que foram encontrados foi de grande valia, pois foi constatado que os efeitos mais influentes no estudo foram a temperatura, que está relacionado com o aumento da energia de ativação, e o tempo de reação, que está relacionado com o equilíbrio da reação. A quantidade de catalisador na reação mostrou ter pouca significância no diagrama de Pareto. Entretanto, é de fundamental importância no meio reacional, pois sem o catalisador não há boa conversão. Por outro lado, um excesso demasiado não aumenta a conversão na mesma proporção, podendo até prejudicar o processo em algum momento (gráfico de superfície de resposta). De acordo com MSR, tem-se que os pontos que levam ao máximo de conversão para o catalisador Amberlyst ${ }^{\circledR}$ 15, 95,745 \%, correspondem a uma quantidade de catalisador de $7,344 \%$ em peso, temperatura de reação de $99,005^{\circ} \mathrm{C}$ e tempo reacional de 338,16 minutos. Os resultados obtidos neste trabalho mostraram que o processo de produção do bioquerosene com os ácidos graxos livres de 6 átomos a 16 átomos de carbonos proposto foi apropriado para a obtenção do biocombustível, pois o bioproduto apresentou características similares, quando comparado aos dados encontrados para o querosene fóssil de aviação comercial, alguma propriedade do bioquerosene que ainda esteja fora de especificação, pode ser facilmente enquadrada ao realizar a mistura deste em proporções adequadas com o combustível de aviação comercial, fato este que torna o biocombustível promissor.

\section{AGRADECIMENTOS}

Esta pesquisa foi apoiada pela Universidade Federal do Ceará (UFC), Laboratório de Imagem Central Analítica da UFC, Fundação Núcleo de Tecnologia Industrial do Ceará (NUTEC) e financiada pelo Conselho Nacional de Desenvolvimento Científico e Tecnológico (CNPq 478488/2013-0) e pela Fundação Cearense de Apoio ao Desenvolvimento Científico e Tecnológico (FUNCAP).

\section{REFERÊNCIAS}

ASTM standard D1655-13a, "Standard specification for aviation turbine fuels" ASTM International, 2014.

AVRAMOVIC, J. M.; VELICKOVIC, A. V.; STAMENKOVIC, O. S.; RAJKOVIC, K. M.; MILIC, P. S.; VELJKOVIC, V. B. Optimization of sunflower oil ethanolysis catalyzed by calcium oxide: RSM versus ANN-GA. Energy Conversion and Management, v.105, p.1149-1156, 2015.

BOZ, N.; DEGIRMENBASI, N.; KALYON, D. M. Esterification and transesterification of waste cooking oil over Amberlyst 15 and modified Amberlyst 15 catalysts. Applied Catalysis B: Environmental, v.165, p.723-730, 2015. 
CHAI, M.; TU, Q.; LU, M.; YANG, Y. J. Esterification pretreatment of free fatty acid in biodiesel production from laboratory to industry. Fuel Processing Technology, v.125, p.106-113, 2014.

CHAROENCHAITRAKOOL, M.; THIENMETHANGKOON, J. Statistical optimization for biodiesel production from waste frying oil through two-step catalyzed process. Fuel Processing Technology, v.92, n.1, 112-118, 2011.

CIOPEC, M.; DAVIDESCU, C. M.; NEGREA, A.; LUPA, L.; POPA, A.; MUNTEAN, C.; ARDELEAN, R.; ILIA, G. Synthesis, characterization, and adsorption behavior ofaminophosphinic grafted on poly(styrene-co-divinylbenzene) for divalent metalions in aqueous solutions. Polymer Engineering and Science, v. 53, n. 5, 1117-1124, 2013.

COSTA, J. F.; ALMEIDA, M. F.; ALVIN-FERRAZ, M. C. M.; DIAS J. M. Biodiesel production using oil from fish canning industry wastes. Energy Conversion and Management, v.74, p.17-23, 2013.

CVENGROS, J. e CVENGROSOVA, Z. Used frying oils and fats and their utilization in the production of methyl esters of higher fatty acids. Biomass and Bioenergy, v.27, p.173$181,2004$.

DE LIMA, A. P.; DE LIMA A. L.; SANTOS, D. Q.; NETO, W. B. Application of factorial and response surface methods to optimize ethyl biodiesel production from corn oil. Revista Virtual de Química, v.5, n.5, p.817-827, 2013.

DONG, S.; BORTNER, M. J.; ROMAN, M. Analysis of the sulfuric acid hydrolysis of wood pulp for cellulose nanocrystal production: a central composite design study. Industrial Crops and Products, v.93,p.76-87, 2016.

FAN, G.; LIAO, C.; FANG, T.; LUO, S.; SONG, G. Amberlyst 15 as a new and reusable catalyst for the conversion of cellulose into cellulose acetate. Carbohydrate Polymers, v. 112, 203-209, 2014.

FELIZARDO, P.; CORREIA, M. J. N.; RAPOSO, I.; MENDES, J. F.; BERKEMEIER, R.; BORDADO, J. M. Production of biodiesel from waste frying oils. Waste Management, v.26, 487-494, 2006.

GAN, S.; NG, H. K.; CHAN, P. H.; LEONG, F. L. Heterogeneous free fatty acids esterification in waste cooking oil using ion-exchange resins. Fuel Processing Technology, v.102, 67-72, 2012.

HONG, T. D.; SOERAWIDJAJA, T. H.; REKSOWARDOJO, I. K.; FUJITA, O.; DUNIANI, Z.; PHAM, M. X. A study on developing aviation biofuel for the tropics: production process - experimental and theoretical evaluation of their blends with fóssil kerosene. Chemical Engineering and Processing: Process Intensification, v.74, p.124-130, 2013.

IATA - International Air Transport Association. A global approach to reducing aviation emissions - First stop: carbon-neutral growth by 2020. June 2009. Disponível em: http://corporate.airfrance.com/fileadmin/dossiers/img_rte_fr/IATA.pdf. Acesso em: 13 abr. 2016.

INSTITUTO ADOLFO LUTZ. Métodos físico-químicos para análises de alimentos. 4nd ed. São Paulo (Brasil): IMESP. Capítulo XVI, Óleos e gorduras; p. 589-625, 2005.

KUMAR, V.; MUTHURAJ, M.; PALADHANVI, B.; GHOSHAL, A. K.; DAS D. Evaluation and optimization of two stage sequential in situ transesterification process for fatty acid methyl ester quantification from microalgae. Renewable Energy, v.68, p.560-569, 2014. 
LI, L.; CHENG, J.; YANG, Z. R. Preparation and slow relase behavior of porous hydroquinone-carrying poly (styrene-divinylbenzene) microshperes. Journal os Materials Science and Engineering, v. 27, n. 3, 337-343, 2009.

LIU, W.; YIN, P.; ZHANG, J.; TANG, Q.; QU, R. Biodiesel production from esterification of free fatty acid over PA/NaY solid catalyst. Energy Conversion and Management, v.82, p.83-91, 2014.

LLAMAS, A.; AL-LAL, A.; HERNANDEZ, M.; LAPUERTA, M.; CANOIRA, L. Biokerosene from babassu and camelina oils: production and properties of their blends with fóssil kerosene. Energy e Fuels, v.26, p.5968-5976, august. 2012.

PONTE, F. A. F; MACIEL, I. A; LIMA, M. B.; MALVEIRA, J. Q.; ALBUQUERQUE, M. C. G. Estudo da obtenção de bioproduto via catálise heterogênea. Revista SODEBRAS. V.10, n. 111, 116-120, 2015.

QUERINO, V. S. Balanço de massa e energia em uma planta de produção de bioquerosene. 2014. 52 p. Monografia (Graduação em Engenheiro Industrial Químico) - Escola de Engenharia de Lorena, Universidade de São Paulo, Lorena, 2014.

RANUCCI, C. R.; ALVES, H. J.; SILVA, C.; MONTEIRO, M. R.; SANTOS, K. A.; BARICCATTI, R. A.; SILVA, E. A. Obtenção de bioquerosene de pinhão-manso (Jatropha curcas L.) e suas misturas ao querosene fóssil. Revista Tecnológica Edição Especial 2014, Maringá, P.43-52, 2015.

RINCÓN, L. E.; JARAMILLO, J. J.; CARDONA, C. A. Comparison of fesdstocks and technologies for biodiesel production: an environmental and Techno-economic evaluation. Renewable Energy, v.69, p.479-487, 2014.

STACY, C. J.; MELICK, C. A.; CAIRNCROSS, R. A. Esterification of free fatty acids to fatty acid alkyl esters in bubble column reactor for use as biodiesel. Fuel Processing Technology, v.124, p.70-77, 2014

TIWARI, A. K.; KUMA, A.; RAHEMAN, H. Biodiesel production from jatropha oil (Jatropha curcas) with high free fatty acids: an optimized process. Biomass and Bioenergy, v.31, p.569-575, 2007.

WADUD, Z. Decomposing the drivers of aviation fuel demand using simultaneous equation models. Energy, v.83, n.1, p.551-559, 2015.

WAKIL, M. A.; KALAM, M. A.; MASJUKI, H. H.; ATABANI, A. E.; RIZWANUL FATTAH, I. M. Influence of biodiesel blending on physicochemical properties and importance of mathematical model for predicting the properties of biodiesel blend. Energy Conversion and Management, v. 94, p. 51-67, 2015. 


\title{
CENTRAL COMPOSITE DESIGN APPLIED TO BIOQUEROSENE OBTAINING SYSTEM VIA HETEROGENEOUS CATALYSIS
}

\author{
F. A. F. da PONTE' , J. S. RODRIGUES 2 , J. Q. MALVEIRA², I. A. MACIEL ${ }^{3}$, M. C. G. \\ ALBUQUERQUE ${ }^{3}$ \\ ${ }^{1}$ Universidade Federal do Ceará, Departamento de Engenharia Química \\ ${ }^{2}$ Núcleo de Tecnologia Industrial do Ceará, Laboratório de Biocombustível \\ ${ }^{3}$ Universidade Federal do Ceará, Departamento de Engenharia Mecânica \\ E-mail: flaviodaponte@hotmail.com
}

\begin{abstract}
In this work biokerosene was produced by the esterification reaction (using heterogeneous catalyst such as Amberlyst $\left.{ }^{T M} 15\right)$ of free fatty acids of C6 to C16 carbon atoms derived from babassu oil (Orbinya speciosa) using a $2^{3}$ factorial experimental design with central composite. Scanning electron microscopy morphology and the thermogravimetry analysis was used to indicate the working range of the catalyst. The process parameters studied were the reaction temperature between $70{ }^{\circ} \mathrm{C}$ to $100{ }^{\circ} \mathrm{C}$, catalyst concentration between $5 \%$ to $10 \%$ and reaction time between 240 minute to 480 minute. As the factorial design response parameter was evaluated the methyl esters content. The process optimization was performed using the response surface methodology (RSM) and the polynomial model of second order. It was observed that the most influential process variables were the reaction temperature and time, the highest conversion of methyl esters was obtained with $7.5 \%$ catalyst; reaction temperature of $85^{\circ} \mathrm{C}$ and 360 minutes of reaction. The best conversion of the reaction was $94.7 \%$ on biofuel. Utilizing the MRS with second-order polynomial model, the optimized conditions achieved for the esterification reaction with Amberlyst ${ }^{\circledR} 15$ were: a temperature of $90.0^{\circ} \mathrm{C}$, catalyst amount of $7.3 \%$, and reaction time 338.2 minutes. The expected theoretical yield of conversion into free fatty acids from C6 to C16 carbon atoms in biokerosene was $95.7 \%$.
\end{abstract}

KEYWORDS: Central composite design (CCD); Biokerosene; Free fatty acids; Babassu oil; Response surface methodololy (RSM). 